\title{
Schizophrenia and comorbid sleep disorders
}

\author{
Maurits van den Noort ${ }^{1,2}$, Heike Staudte ${ }^{3}$, Benoît Perriard ${ }^{4}$, Sujung Yeo ${ }^{5}$, Sabina Lim ${ }^{1}$, Peggy Bosch ${ }^{3,6}$ \\ ${ }^{1}$ Research Group of Pain and Neuroscience, Kyung Hee University, Seoul 130-701, South Korea \\ ${ }^{2}$ Brussels Institute for Applied Linguistics, Vrije Universiteit Brussel, 1050 Brussels, Belgium. \\ ${ }^{3}$ Psychiatric Research Institute, LVR-Klinik Bedburg-Hau, 47511 Bedburg-Hau, Germany. \\ ${ }^{4}$ Neurology Unit, Laboratory for Cognitive and Neurological Sciences, Department of Medicine, Faculty of Science, University of Fribourg, 1700 Fribourg, Switzerland. \\ ${ }_{5}^{5}$ Department of Acupuncture and Meridian of Oriental Medicine, Sang Ji University, Wonju 26339, South Korea. \\ ${ }^{6}$ Centre for Cognition, Donders Institute for Brain, Cognition and Behaviour, Radboud University Nijmegen, 6500 GL Nijmegen, Gelderland, the Netherlands.
}

Correspondence to: Prof. Maurits van den Noort, Research Group of Pain and Neuroscience, Kyung Hee University, \#47 Gyeonghuidae-Gil, Dongdaemun-Gu, Seoul 130-701, South Korea. E-mail: info@mauritsvandennoort.com

How to cite this article: van den Noort M, Staudte H, Perriard B, Yeo S, Lim S, Bosch P. Schizophrenia and comorbid sleep disorders. Neuroimmunol Neuroinflammation 2016;3:225-7.

Article history: Received: 18-09-2016 Accepted: 20-09-2016 Published: 28-10-2016

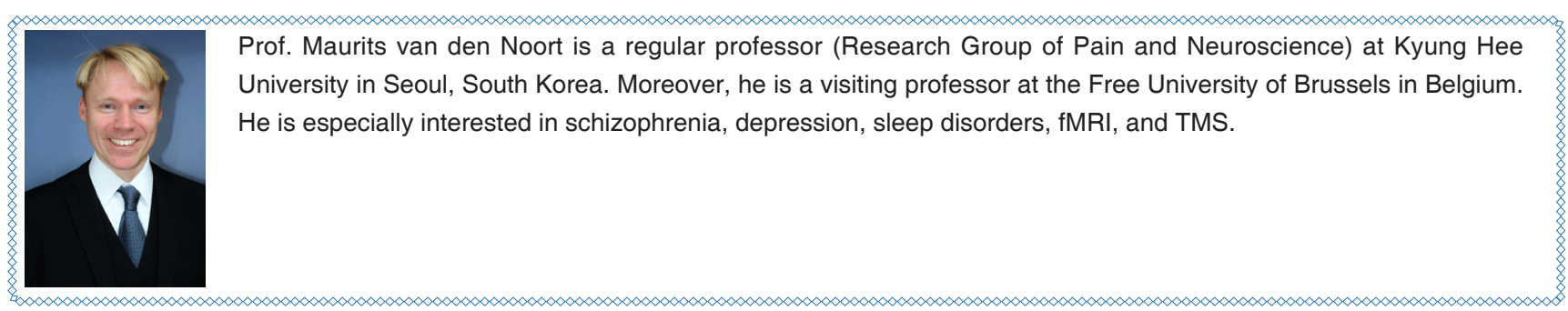

Schizophrenia is a severe psychiatric disorder that has a worldwide prevalence of $0.5 \%{ }^{[1]}$ and poses a high cost to society. ${ }^{[2]}$ The disorder is characterized by positive symptoms, such as hallucinations and delusions, ${ }^{\left[{ }^{3]}\right.}$ negative symptoms, such as impaired emotional functioning and behavioral disruptions (e.g. flat affect, difficulty in starting activities and completing them, etc. $),{ }^{[4]}$ and cognitive symptoms, such as deficits in executive functioning, impaired working memory, and attention problems. ${ }^{[5]}$ Less known to the general public is the fact that a large number of the patients with schizophrenia suffer from sleep disturbances, such as reduced sleep efficiency, reduced total sleep time, and increased sleep latency. ${ }^{[6]}$ Surprisingly, those sleep problems in patients with schizophrenia are also often under-estimated in daily clinical practice. ${ }^{[7]}$

In the Diagnostic and Statistical Manual of Mental Disorders 5th Edition (DSM-V), ${ }^{[8]}$ sleep-wake disorders are classified into 10 disorders or disorder groups (e.g. insomnia disorder, restless legs syndrome, circadian rhythm sleep-wake disorders, etc.). Patients suffering from sleep-wake disorders have problems with respect to the quality, the timing, and the total amount of sleep, ${ }^{\left[{ }^{[8]}\right.}$ leading to distress and impairment in their social and cognitive functioning. ${ }^{[9]}$

Several treatments have been used in patients with 
schizophrenia, as well as patients with sleep-wake disorders. Pharmacological treatment with first- and second-generation antipsychotics (e.g. amisulpride, clozapine, olanzapine, risperidone, etc.) is still the most frequently used treatment in patients with firstepisode and long-term schizophrenia. ${ }^{[10]}$ However, mainly because of the adverse effects of the pharmacological treatment, non-pharmacological addon treatments, such as cognitive behavioral therapy, ${ }^{[11]}$ are being increasingly used. Sleep-wake disorders are mostly treated with pharmacological interventions, such as benzodiazepines, zolpidem, zaleplon, etc., but unfortunately, side-effects are common here, as well. ${ }^{[12]}$ In addition, non-pharmacological interventions, such as cognitive psychotherapy, sleep hygiene, relaxation therapy, acupuncture, etc. ${ }^{[13,14]}$ are used.

Previous research involving patients with schizophrenia and comorbid sleep disorders has shown that a relation exists between sleep problems and cognitive functioning. ${ }^{[15]}$ For instance, in a recent study, a significant negative relationship was found between the number of sleep problems and the working memory performance; i.e. the more severe the patient's sleep problems was, the lower the patient's working memory performance was. ${ }^{[15]}$ However, more research is warranted, and to date, many questions remain unanswered: Firstly, how are poor sleep and decreased social and cognitive functioning in patients with schizophrenia related? Secondly, what role does the pharmacological treatment of patients with schizophrenia play in their impaired sleep and social and cognitive functioning? For instance, benzodiazepines are known to suppress rapid-eyemovement (REM) sleep ${ }^{[12]}$ and when patients stop such medications, episodes of increased REM sleep are more numerous. ${ }^{[12]}$ Because REM sleep plays a role in the learning process, as well as in memory consolidation, ${ }^{[12]}$ future research should clarify whether benzodiazepines might have a negative influence on cognitive functioning, e.g. working memory, in patients with schizophrenia and comorbid sleep disorders. Thirdly, how does the pharmacological treatment of comorbid sleep disorders in patients with schizophrenia interfere with the pharmacological treatment of the positive and the negative symptoms of those patients? These questions need to be investigated and answered in future studies so as to improve further the treatment and the quality of life of patients with schizophrenia and comorbid sleep disorders.

Here, an important finding is that in previous research, patients with schizophrenia tended to underestimate their problems on self-report inventories. ${ }^{[16]}$ Therefore, in future research, both "objective" (e.g. electroencephalography, actiwatches, etc.) and "subjective" (e.g. self-report inventories, such as the Pittsburgh Sleep Quality Index, ${ }^{[17]}$ Munich Parasomnia Screening, ${ }^{[18]}$ etc.) measurements must be used if the efficiencies of various pharmacological and non-pharmacological treatments of patients with schizophrenia and comorbid sleep disorders are to be determined with accuracy.

To conclude, many patients with schizophrenia suffer from comorbid sleep-wake disorders. Therefore, in daily clinical practice, sleep needs more attention in the treatment of patients with schizophrenia so that such patients receive optimal treatment and their qualities of life are increased. Finally, evidence for adding "disturbed sleep" as one of the characteristic symptoms of schizophrenia in the DSM system seems to be mounting. ${ }^{[8,15]}$

\section{Financial support and sponsorship Nil.}

\section{Conflicts of interest \\ There are no conflicts of interest.}

\section{Patient consent \\ No patient involved.}

\section{Ethics approval}

This article does not contain any studies with human participants or animals.

\section{REFERENCES}

1. Messias EL, Chen CY, Eaton WW. Epidemiology of schizophrenia: review of findings and myths. Psychiatr Clin North Am 2007;30:323-38.

2. Evensen S, Wisløff T, Lystad JU, Bull H, Ueland T, Falkum E. Prevalence, employment rate, and cost of schizophrenia in a highincome welfare society: a population-based study using comprehensive health and welfare registers. Schizophr Bull 2016;42:476-83.

3. Patel KR, Cherian J, Gohil K, Atkinson D. Schizophrenia: overview and treatment options. P T 2014;39:638-45.

4. Gur RE, Kohler CG, Ragland JD, Siegel SJ, Lesko K, Bilker WB, Gur RC. Flat effect in schizophrenia: relation to emotion processing and neurocognitive measures. Schizophr Bull 2006;32:279-87.

5. Orellana G, Slachevsky A. Executive functioning in schizophrenia. Front Psychiatry 2013;4:35.

6. Cohrs S. Sleep disturbances in patients with schizophrenia: impact and effect of antipsychotics. CNS Drugs 2008;22:939-62.

7. Bosch P, editor. Needles on the couch: acupuncture in the treatment of depression, schizophrenia and sleep disorders. Nijmegen: Vantilt Uitgeverij; 2015.

8. American Psychiatric Association. Diagnostic and Statistical Manual of Mental Disorders, 5th ed. Arlington, VA: American Psychiatric Publishing; 2013.

9. Szentkirályi A, Madarász CZ, Novák M. Sleep disorders: impact on daytime functioning and quality of life. Expert Rev Pharmacoecon Outcomes Res 2009;9:49-64.

10. Kane JM, Correll CU. Pharmacologic treatment of schizophrenia 
Dialogues Clin Neurosci 2010;12:345-57.

11. Keshavan MS, Roberts M, Wittmann D. Guidelines for clinical treatment of early course schizophrenia. Curr Psych Rep 2006;8:329-34.

12. Pagel JF, Parnes BL. Medications for the treatment of sleep disorders: an overview. Prim Care Companion J Clin Psychiatry 2001;3:118-25.

13. Harsora P, Kessmann J. Nonpharmacologic management of chronic insomnia. Am Fam Physician 2009;79:125-30.

14. Cao H, Pan X, Li H, Liu J. Acupuncture for treatment of insomnia: a systematic review of randomized controlled trials. $J$ Altern Complement Med 2009;15:1171-86.

15. Van den Noort M, Struys E, Perriard B, Staudte H, Yeo S, Lim S, Bosch P. Schizophrenia and depression: the relation between sleep quality and working memory. Asian J Psychiatr 2016;24:73-8.

16. Bosch P, Van Luijtelaar G, Van den Noort M, Schenkwald J, Kueppenbender N, Lim S, Egger J, Coenen A. The MMPI-2 in chronic psychiatric illness. Scand J Psychol 2014;55:513-9.

17. Buysse DJ, Reynolds CF, Monk TH, Berman SR, Kupfer DJ. The Pittsburgh Sleep Quality Index: a new instrument for psychiatric practice and research. Psychiatry Res 1989;28:193-213.

18. Fulda S, Hornyak M, Müller K, Cerny L, Beitinger PA, Wetter TC Development and validation of the Munich Parasomnia Screening (MUPS): a questionnaire for parasomnias and nocturnal behaviors. Somnologie 2008;12:56-65. 